\title{
Revisão taxonômica de Termitococcinae (Hemiptera, Coccoidea, Margarodidae)
}

\author{
Daniele C. da Silva ${ }^{1} \&$ Gervásio S. Carvalho ${ }^{2}$
} 1. Fundação Estadual de Pesquisa Agropecuária (FEPAGRO), Laboratório de Entomologia e Museu Ramiro Gomes Costa. Rua Gonçalves Dias, 570, $90130-060$ Porto Alegre, RS, Brasil.
(danientomo@yahoo.com.br)
2. Pontifícia Universidade Católica do Rio Grande do Sul, Faculdade de Biociências, Departamento de Biodiversidade e Ecologia, Laboratório de Entomologia. Av. Ipiranga, 6681, 90619-900
Porto Alegre, RS, Brasil. (gervasio@pucrs.br)

\begin{abstract}
Taxonomic revision of the Termitococcinae (Hemiptera, Coccoidea, Margarodidae). The species of Termitococcinae are revised. This subfamily, restricted to the Neotropical region, is composed of five valid species included in two genera: Termitococcus aster Silvestri, 1901, Termitococcus carratoi Silvestri, 1936, Eurhizococcus brasiliensis (Wille, 1922), Eurhizococcus brevicornis (Silvestri, 1901), and Eurhizococcus colombianus Jakubski, 1965. The present study provides the redescription of these species, including the morphological characterization of the three stages (crawlers, cyst and adult female). Identification keys and illustrations for the species are also included. Lectotypes and paralectotypes of two species are designated.
\end{abstract}

KEYWORDS. Taxonomy, scale insect, ground pearls, Neotropical.

RESUMO. As espécies de Termitococcinae são revisadas. Esta subfamília, restrita à Região Neotropical, é composta de cinco espécies válidas, incluídas em dois gêneros: Termitococcus aster Silvestri, 1901, Termitococcus carratoi Silvestri, 1936, Eurhizococcus brasiliensis (Wille, 1922), Eurhizococcus brevicornis (Silvestri, 1901) e Eurhizococcus colombianus Jakubski, 1965. O estudo traz a redescrição das espécies da subfamília, incluindo a caracterização morfológica dos três estágios de desenvolvimento (ninfa ambulatória, cisto e fêmea adulta). Chaves de identificação e ilustrações são também incluídas. São designados lectótipos e paralectótipos para duas espécies.

PALAVRAS-CHAVE. Taxonomia, cochonilha, pérolas-da-terra, Neotropical.

Margarodidae sensu stricto constitui um grupo de cochonilhas peculiar. São denominadas pérolas-da-terra, pois vivem no solo alimentando-se na raiz das plantas hospedeiras, sob a forma de um cisto durante a maior parte de seu desenvolvimento.

Entre as características que distinguem as fêmeas adultas podemos destacar o corpo com cerca de cinco milímetros, arredondado ou alongado, coberto por cerdas longas ("hair-like"); as pernas anteriores fossoriais, adaptadas para cavar no solo; e a ausência de peças bucais e olhos (Morrison, 1928; GRANARA DE WillinK, 1995; Foldi, 2005; Miller et al., 2007).

JAKUBSKI (1965) dividiu a família em duas, Margarodidae e Termitococcidae, sendo Eurhizococcus Silvestri, 1936 e Termitococcus Silvestri, 1901 pertencentes a esta última. Nesta mesma ocasião, propôs Termitococcinae constituída por duas tribos, Termitococcini e Eurhizococcini.

FoLDI (2005) restabeleceu os dois grupos em uma única família, Margarodidae, e distribuiu as 105 espécies em seis subfamílias: Margarodinae, Margarodesiinae, Eumargarodinae, Neomargarodinae, Porphyrophorinae e Termitococcinae.

$\mathrm{Na}$ Região Neotropical, os margarodídeos estão representados por 12 espécies, incluídas em Margarodinae e Termitococcinae. No Brasil são conhecidas: Margarodes carvalhoi Costa Lima, 1950; Margarodes paulistus Silvestri, 1939 e Margarodes vitis (Philippi, 1884) (Margarodinae) e Eurhizococcus brasiliensis (Wille, 1922), Eurhizococcus brevicornis (Silvestri, 1901) e Termitococcus carratoi (Silvestri, 1936) (Termitococcinae) (Silva et al., 1968; Foldi, 2001, 2005).
Termitococcinae é exclusivamente neotropical e as fêmeas adultas caracterizam-se principalmente por possuírem corpo alongado e coberto intensamente por cerdas de diferentes tamanhos. As cinco espécies são distribuídas em dois gêneros: Termitococcus aster Silvestri, 1901 e T. carratoi (Termitococcini) e $E$. brasiliensis, E. brevicornis e Eurhizococcus colombianus Jakubski, 1965 (Eurhizococcini).

Com o objetivo de contribuir com a taxonomia do grupo, é apresentada a revisão de Termitococcinae, com a inclusão de chaves, redescrições das espécies e ilustrações.

\section{MATERIAL E MÉTODOS}

Foram examinados exemplares das seguintes instituições: Dipartimento di Entomologia e Zoologia Agraria, Università degli Studi di Napoli "Federico II", Portici, Itália (IFSP); Museu de Zoologia, Universidade de São Paulo, São Paulo, Brasil (MZSP); Museu Ramiro Gomes Costa, Fundação Estadual de Pesquisa Agropecuária do Rio Grande do Sul, Porto Alegre, Brasil (MRGC); The Bohart Museum of Entomology, University of California, Davis, USA (UCDC).

O material estudado foi preparado e examinado no Laboratório de Entomologia da Pontifícia Universidade Católica do Rio Grande do Sul (PUCRS) e na Fundação Estadual de Pesquisa Agropecuária (FEPAGRO), em Porto Alegre, RS, com auxílio de microscópio óptico, estereomicroscópio e microscópio eletrônico de varredura.

$\mathrm{Na}$ preparação das lâminas permanentes foi adotada a técnica de Granara de Willink (1990), 
utilizando-se para corar a solução de Essig, em substituição ao ácido acético glacial. Depois de corados os exemplares passaram por uma série alcoólica de diferentes concentrações, $70 \%$ e $96 \%$, posteriormente imersos em óleo de cravo, e finalmente montados em lâminas com Bálsamo do Canadá. As lâminas permanentes foram incorporadas à coleção de Coccoidea do MRGC e do Museu de Ciências e Tecnologia da PUCRS (MCTP).

Para as medidas dos exemplares foi utilizada ocular micrométrica acoplada aos microscópios estereoscópico e óptico.

Na lista de hospedeiros e distribuição geográfica, foram utilizados os dados de procedência contidos nas etiquetas dos espécimes, além daqueles encontrados na bibliografia.

Chave para as espécies de Termitococcinae, com base nos caracteres da ninfa ambulatória (primeiro instar)

1. Corpo alongado, com número reduzido de cerdas dorsais e ventrais distribuídas esparsamente; sete pares de espiráculos abdominais; antena com quatro artículos Margarodinae

1'. Corpo circular, coberto por cerdas mais concentradas nas regiões marginal e submarginal; cerdas caudais presentes ou ausentes; sete ou oito pares de espiráculos abdominais; antenas com um a três artículos

Termitococcinae 2

2. Oito pares de espiráculos abdominais; antena com três artículos, sendo o último cerca de 8 a 12 vezes maior que os demais

Termitococcus 3

2'. Sete pares de espiráculos abdominais; corpo com cerdas longas, com até $425 \mu \mathrm{m}$ de comprimento; antena com um ou dois artículos, o último cerca de nove vezes maior que o primeiro Eurhizococcus 4

3. Tíbia e tarso fusionados dorsalmente

Termitococcus aster

3'. Tíbia e tarso não fusionados dorsalmente Termitococcus carratoi

4. Antenas com dois artículos; um par de cicatrizes metatorácicas formando um eixo paralelo em relação às pernas metatorácicas ..........Eurhizococcus colombianus 4'. Antenas com um artículo 5

5. Um par de cicatrizes metatorácicas formando um eixo perpendicular em relação às pernas metatorácicas

Eurhizococcus brevicornis

5'. Dois pares de cicatrizes metatorácicas, não formando um eixo perpendicular em relação às pernas metatorácicas Eurhizococcus brasiliensis

Chave para as espécies de Termitococcinae, com base nos caracteres da fêmea adulta

1. Corpo oval, com cerdas longas e espiniformes distribuídas esparsamente na face ventral, exceto nas regiões marginal e submarginal, onde são mais concentradas; sete pares de espiráculos abdominais; antenas com sete ou oito artículos.............. Margarodinae 1'. Corpo alongado, densamente coberto por cerdas curtas e longas; sete ou oito pares de espiráculos abdominais; antenas com cinco ou seis artículos. Termitococcinae 2

2. Oito pares de espiráculos abdominais; corpo com cerdas de cerca de $900 \mu \mathrm{m}$ de comprimento, densamente distribuídas ventral e dorsalmente

Termitococcus carratoi 2'. Sete pares de espiráculos abdominais; corpo com cerdas longas ("hair-like") densamente distribuídas pelo corpo, com cerca de $1,1 \mathrm{~mm}$ de comprimento ............... 3 3. Antenas com cinco artículos.

Eurhizococcus brasiliensis 3'. Antenas com seis artículos

Eurhizococcus colombianus

Termitococcus Silvestri, 1901

Termitococcus SiLVESTRI, 1901:4.

Espécie-tipo: Termitococcus aster Silvestri, 1901, designado por Fernald, 1903:122.

Corpo da ninfa ambulatória circular, cercado por cerdas; antena com três artículos, sendo o último muito longo; olhos ausentes; fêmur longo, tarso uniarticulado (SilVestri, 1901).

Peças bucais desenvolvidas com longos estiletes. Pernas semelhantes em forma e tamanho. Quatro pares de longas cerdas caudais. Oito pares de espiráculos abdominais.

Discussão. Este gênero foi descrito com base nos caracteres da ninfa ambulatória (primeiro instar). Nesse estágio, difere de Eurhizococcus principalmente por apresentar antena com três artículos, oito pares de espiráculos abdominais e cicatrizes metasternais ausentes.

\section{Termitococcus aster Silvestri, 1901}

(Figs 1,2)

Termitococcus aster SILVESTRI, 1901:4.

Termiticoccus (sic) aster; MACGILLIVRAY, 1921:141.

Redescrição. Ninfa ambulatória ( $1^{\circ}$ instar). Corpo circular, com cerca de $755 \mu \mathrm{m}$ de comprimento e $720 \mu \mathrm{m}$ de largura e coberto por cerdas de diferentes tamanhos; poros ausentes. Ventre: antena com três artículos - I, $17 \mu \mathrm{m}$; II, $19 \mu \mathrm{m}$; III, $167 \mu \mathrm{m}$-, este último cerca de 8 a 9 vezes maior que os demais, com extremidade afilada e sete cerdas curtas (Fig. 1). Olhos ausentes. Longas cerdas em toda a margem do corpo, que podem alcançar até $215 \mu \mathrm{m}$. Cerdas do corpo em menor número e mais curtas, com até $75 \mu \mathrm{m}$. Peças bucais desenvolvidas, estiletes longos enrolados entre as pernas podendo dar até duas voltas completas (Fig. 2). Pernas desenvolvidas e semelhantes, em forma e tamanho: coxa globosa; trocanter com duas ou três 
cerdas curtas $(15 \mu \mathrm{m})$; fêmur longo e robusto, com cinco cerdas; tíbia e tarso delgados, fusionados dorsalmente; tíbia com uma única cerda ventral próxima ao tarso, com $35 \mu \mathrm{m}$; tarso uniarticulado, menor que a tíbia, com dois digítulos na extremidade, próxima à unha; unha levemente curvada. Pernas protorácicas: coxa $68 \mu \mathrm{m}$; trocanter, $28 \mu \mathrm{m}$; fêmur, $180 \mu \mathrm{m}$ de comprimento e $58 \mu \mathrm{m}$ de largura; tíbia, $105 \mu \mathrm{m}$; tarso, $58 \mu \mathrm{m}$; unha, $45 \mu \mathrm{m}$ (Fig. 1). Pernas mesotorácicas: coxa, $74 \mu \mathrm{m}$; trocanter, $25 \mu \mathrm{m}$; fêmur, $180 \mu \mathrm{m}$ de comprimento e 55 $\mu \mathrm{m}$ de largura; tíbia, $105 \mu \mathrm{m}$; tarso, $70 \mu \mathrm{m}$; unha, 52 $\mu \mathrm{m}$. Pernas metatorácicas: coxa, $73 \mu \mathrm{m}$; trocanter, 27 $\mu \mathrm{m}$; fêmur, $175 \mu \mathrm{m}$ de comprimento e $54 \mu \mathrm{m}$ de largura; tíbia, $110 \mu \mathrm{m}$; tarso, $80 \mu \mathrm{m}$; unha, $40 \mu \mathrm{m}$. Quatro pares de cerdas caudais com 360 a $410 \mu \mathrm{m}$. Dorso: oito pares de espiráculos abdominais. Cerdas com 115 a $185 \mu \mathrm{m}$ de comprimento, mais concentradas nas regiões da cabeça e do tórax. Ânus esclerotizado em forma de U.

Ninfa fixa $\left(2^{\circ}\right.$ instar - Cisto). Desconhecida.

Fêmea adulta. Desconhecida.

Material examinado. Para preservar a estabilidade da nomenclatura desta espécie, um lectótipo é aqui designado entre os síntipos. Lectótipo, PARAGUAI, Alto Paraná: Tucuru Pucu, (ninfa ambulatória), espécime em lâmina (IFSP). Paralectótipos, (ninfas), 3 espécimes em 2 lâminas com os mesmos dados do lectótipo.

Distribuição geográfica. Paraguai (Alto Paraná).

Biologia. Encontrada dentro do termiteiro de Leucotermitis tenuis (Hag.) (JAKUBSKI, 1965; BEN-Dov, 2005).

\section{Termitococcus carratoi Silvestri, 1936 (Figs 3-5)}

Termitococcus carratoi Silvestri, 1936:34.

Redescrição. Ninfa ambulatória ( $1^{\circ}$ instar). Corpo circular com 1,2 mm de comprimento e 1,0 $\mathrm{mm}$ de largura e coberto por cerdas de diferentes tamanhos; ausência de poros. Ventre: antena com três artículos - I, $21 \mu \mathrm{m}$; II, 15 $\mu \mathrm{m}$; III, $183 \mu \mathrm{m}$-, este último 9 a 12 vezes mais longo que os demais, afilado na extremidade e com sete cerdas curtas. Olhos ausentes. Longas cerdas em toda a margem do corpo, com até $210 \mu \mathrm{m}$ de comprimento. Cerdas do corpo mais curtas, escassas e esparsas, com 55 a $60 \mu \mathrm{m}$ de comprimento. Peças bucais desenvolvidas. Pernas desenvolvidas e semelhantes em forma e tamanho: coxa globosa com 85 a $108 \mu \mathrm{m}$ contendo duas cerdas; trocanter com 32 a $39 \mu \mathrm{m}$ e uma cerda muito pequena; fêmur longo e robusto, 212 a $225 \mu \mathrm{m}$ de comprimento e 81 a $86 \mu \mathrm{m}$ de largura contendo três cerdas; tíbia delgada, com 121 a $135 \mu \mathrm{m}$ e uma única cerda; tarso delgado, uniarticulado, com 81 a $95 \mu \mathrm{m}$ e dois digítulos; unha levemente curvada, 61 a $63 \mu \mathrm{m}$. Dorso: oito pares de espiráculos abdominais. Ânus esclerotizado em forma de U.

Ninfa fixa $\left(2^{\circ}\right.$ instar - Cisto). Corpo oval, com 6,0 $\mathrm{mm}$ de comprimento e 3,5 $\mathrm{mm}$ de largura. Ventre: antena pequena e circular com cerca de oito cerdas pequenas. Olhos e pernas ausentes. Peças bucais desenvolvidas. Pequenas cicatrizes espalhadas por todo corpo, principalmente ao redor das peças bucais. Cinco poros periespiraculares presentes nos espiráculos torácicos anteriores e posteriores. Conjunto de pequenas cerdas em torno dos espiráculos torácicos. Dorso: oito pares de espiráculos abdominais. Ânus esclerotizado em forma de U, rodeado por uma placa anal oval (Fig. 3). Conjunto de pequenas cerdas em torno da placa anal e em cada um dos espiráculos abdominais.

Fêmea adulta. Corpo alongado, com 9,5 mm de comprimento e 5,0 $\mathrm{mm}$ de largura e ausência de poros multiloculares. Ventre (Fig. 4): antena com cinco artículos - I, II e III, $70 \mu \mathrm{m}$ cada, IV, $108 \mu \mathrm{m}$ e V oval e mais longo que os demais, com $222 \mu \mathrm{m}$ de comprimento. Corpo coberto densamente por cerdas curtas, médias e longas. Cerdas marginais mais longas que podem alcançar até $900 \mu \mathrm{m}$. Ausência de olhos e peças bucais. Pernas bem desenvolvidas e muito esclerotizadas. Pernas protorácicas do tipo fossorial, com fêmur grande e robusto, trocanter e fêmur fusionados com $1,1 \mathrm{~mm}$ de comprimento e $835 \mu \mathrm{m}$ de largura, coberto por inúmeras cerdas; tíbia + tarso + unha com $980 \mu \mathrm{m}$ de comprimento, segmentação visível, mas destituída de articulação entre os segmentos, dando a impressão que são fusionados; unha curvada. Pernas mesotorácicas e metatorácicas semelhantes, bem menores que as protorácicas: coxa com $250 \mu \mathrm{m}$ de comprimento e $800 \mu \mathrm{m}$ de largura; trocanter e fêmur fusionados, com $570 \mu \mathrm{m}$ de comprimento e 370 $\mu \mathrm{m}$ de largura; tíbia + tarso + unha $850 \mu \mathrm{m}$, destituídos de articulação; unha curvada com dois digítulos. Dorso (Fig. 5): oito pares de espiráculos abdominais (somente o último par ventral), sendo o primeiro par submarginal. Do terceiro ao sétimo segmentos abdominais, pequenas regiões centrais com a derme engrossada, que formam manchas circulares. Corpo densamente coberto por cerdas curtas. Ânus esclerotizado em forma de U.

Material examinado. Lectótipo ㅇ, BRASIL, Mato Grosso do Sul: Três Lagoas, espécime em lâmina (IFSP). Paralectótipos: mesmos dados do lectótipo, 3 espécimes em 2 lâminas (uma lâmina com uma ninfa ambulatória e a outra com dois cistos).

Distribuição geográfica. Brasil (Mato Grosso do Sul). Hospedeiro. Poaceae (JAKUBSKI, 1965; BeN-Dov, 2005; FolDI, 2005).

\section{Eurhizococcus Silvestri, 1936}

Eurhizococcus SiLVESTRI, 1936:39.

Eurrhizococcus; LINDINGER, 1943:219 (erro na grafia).

Eurchizococcus; Costa Lima, 1950:241 (erro na grafia).

Espécie-tipo: Margarodes brasiliensis Wille, 1922, por designação original.

Fêmea adulta com sete pares de espiráculos abdominais, corpo com cerdas longas ("hair-like") densamente distribuídas, poros multiloculares de um 


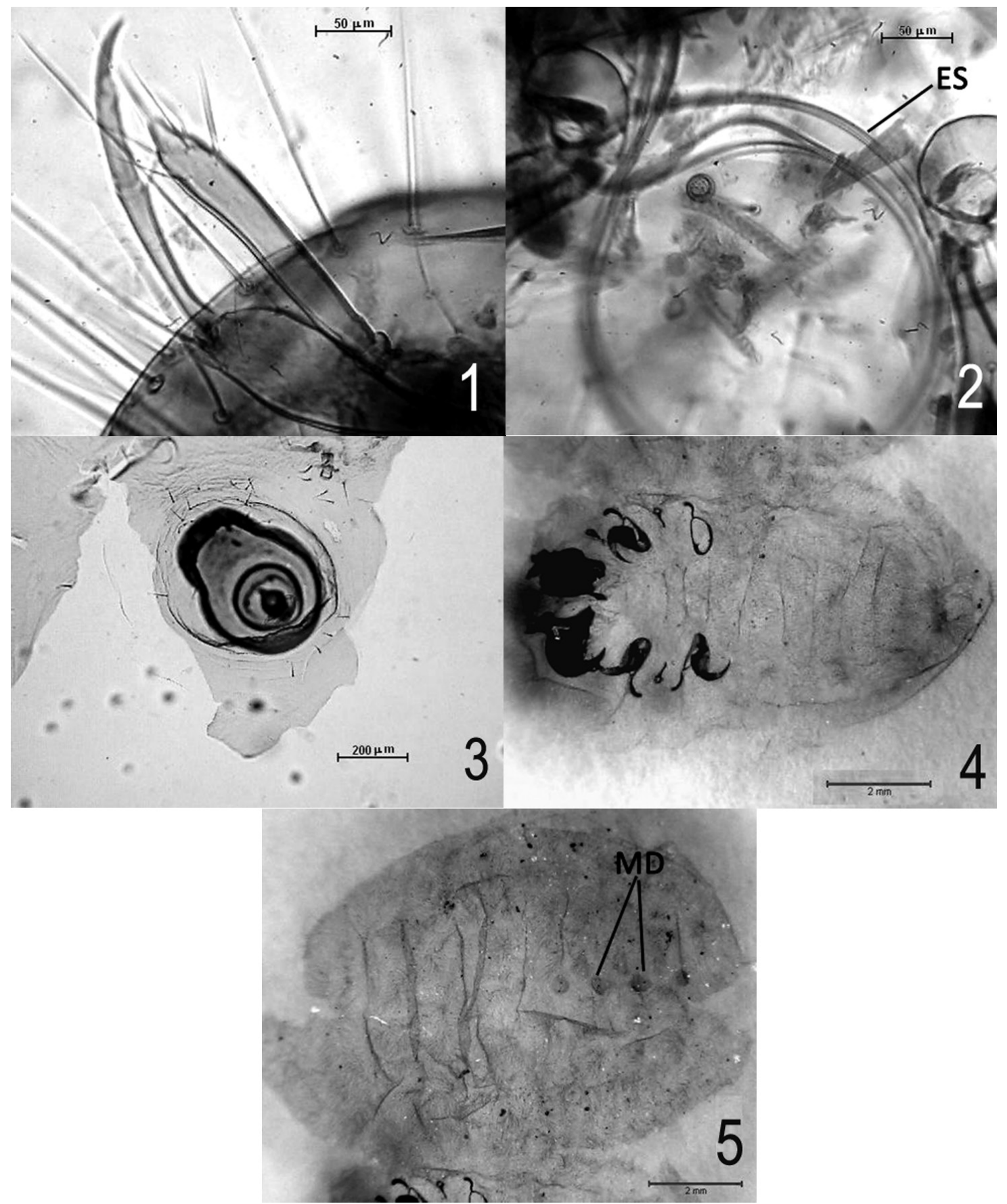

Figs 1-5. Termitococcus aster, ninfa ambulatória: 1, antena e perna protorácica direita; 2, estiletes; Termitococcus carratoi: 3, placa anal do cisto; O: 4 , vista ventral; 5 , vista dorsal, mancha dorsal (ES, estiletes; MD, mancha dorsal).

único tipo com dois anéis: o mais externo com 15 a 20 lóculos e no mais interno quatro a oito lóculos; antena com cinco ou seis artículos.

Ninfa ambulatória circular, rodeada por cerdas longas; antena com um ou dois artículos; presença de cicatrizes metasternais.

\section{Eurhizococcus brasiliensis (Wille, 1922) (Figs 6-11)}

Margarodes brasiliensis WiLLE, 1922:83; Costa Lima, 1924:136. Eurhizococcus brasiliensis; SILVESTRI, 1936:39, 40; JAKUBSKI, 1965:174.
Euchizococcus brasiliensis Costa Lima, 1950:241 (erro de grafia). Margarodes soriai FoldI, 1987:156 (sinonimizado por FoldI, 1989:320)

Material-tipo. Síntipo + (não examinado), BRASIL, Rio Grande do Sul: Santa Maria, 1921, ex. raízes de videira e Petroselinum (Ben-Dov, 2005).

Redescrição. Ovo esbranquiçado, $700 \mu \mathrm{m}$ de comprimento e $450 \mu \mathrm{m}$ de largura.

Ninfa ambulatória ( $1^{\circ}$ instar). Corpo circular, 730 a $950 \mu \mathrm{m}$ de comprimento e 710 a $910 \mu \mathrm{m}$ de 
largura, coberto por cerdas de diferentes tamanhos; poros ausentes. Ventre: antena uniarticulada com 55 a $76 \mu \mathrm{m}$ e oito cerdas de vários tamanhos (Fig. 6). Olhos ausentes. Longas cerdas marginais com até $120 \mu \mathrm{m}$ de comprimento. Cerdas do corpo com $40 \mu \mathrm{m}$, as da região cefálica um pouco mais longas com até $55 \mu \mathrm{m}$ de comprimento. Três pares de cerdas caudais com até $400 \mu \mathrm{m}$ de comprimento. Peças bucais desenvolvidas com longos estiletes enrolados, podem completar duas voltas. Pernas desenvolvidas e semelhantes: coxa globosa; trocanter com duas sensilhas e uma cerda; fêmur longo e robusto, alargado próximo à tíbia, com seis cerdas; tíbia com uma única cerda similar a um grande espinho; tarso uniarticulado com um par de digítulos; unha levemente curvada. Pernas protorácicas: coxa, $65 \mu \mathrm{m}$; trocanter, $27 \mu \mathrm{m}$; fêmur, $155 \mu \mathrm{m}$ de comprimento e $55 \mu \mathrm{m}$ de largura; tíbia, 54 $\mu \mathrm{m}$; tarso, $67 \mu \mathrm{m}$; unha, $43 \mu \mathrm{m}$. Pernas mesotorácicas: coxa, $70 \mu \mathrm{m}$; trocanter, $30 \mu \mathrm{m}$; fêmur, $160 \mu \mathrm{m}$ de comprimento e $67 \mu \mathrm{m}$ de largura; tíbia, $63 \mu \mathrm{m}$; tarso, $69 \mu \mathrm{m}$; unha, $50 \mu \mathrm{m}$. Perna metatorácica: coxa, 72 $\mu \mathrm{m}$; trocanter, $30 \mu \mathrm{m}$; fêmur, $160 \mu \mathrm{m}$ de comprimento e $60 \mu \mathrm{m}$ de largura; tíbia, $65 \mu \mathrm{m}$; tarso, $76 \mu \mathrm{m}$; unha, $51 \mu \mathrm{m}$. Dois pares de cicatrizes metasternais com 18 $\mu \mathrm{m}$ cada (Fig. 7). Sete pares de pequenos espiráculos abdominais, o segundo e terceiro maiores que os demais. Dorso: cerdas escassas. Ânus esclerotizado em forma de U.

Ninfa fixa ( $2^{\circ}$ instar - Cisto). Corpo oval, 4 a 7 $\mathrm{mm}$ de comprimento e 3,5 a $5 \mathrm{~mm}$ de largura. Ventre: antena pequena e circular, com $35 \mu \mathrm{m}$ de diâmetro e nove ou dez cerdas. Olhos e pernas ausentes. Peças bucais desenvolvidas. Conjunto de pequenas cicatrizes de cada lado das peças bucais (cerca de 15), distribuídas no centro na região torácica em conjuntos de sete cicatrizes. Poros periespiraculares torácicos presentes: nove ou dez nos anteriores e até seis nos posteriores. Dorso (Fig. 8): sete pares de espiráculos abdominais, o primeiro submarginal e o último um pouco menor que os demais. Ânus em forma de U rodeado por uma placa suboval esclerotizada que forma dois anéis, o mais externo com até $100 \mu \mathrm{m}$ de diâmetro. Inúmeros poros entre o primeiro e segundo anel da placa anal (até 40).

Fêmea adulta. Corpo alongado, com 7 a $11 \mathrm{~mm}$ de comprimento e 3 a $5 \mathrm{~mm}$ de largura, densamente coberto por cerdas (longas, médias e curtas); segmentação claramente visível; presença de poros multiloculares. Ventre (Fig. 9): antena com cinco artículos - I ao IV, $120 \mu \mathrm{m}$ e V o mais longo, 155 $\mu \mathrm{m}$ (Fig. 10). Todos os artículos com muitas cerdas pequenas que formam fileiras próximo às articulações. Olhos e peças bucais ausentes. Pernas desenvolvidas, sendo o primeiro par robusto do tipo fossorial, adaptada para cavar o solo. Pernas protorácicas: trocanter e fêmur fusionados, $700 \mu \mathrm{m}$ de comprimento e $930 \mu \mathrm{m}$ de largura; fêmur grande e robusto; tíbia + tarso + unha
$825 \mu \mathrm{m}$, segmentação visível, mas sem articulação. Pernas meso- e metatorácicas semelhantes em forma e tamanho: coxa, $220 \mu \mathrm{m}$ de comprimento e $470 \mu \mathrm{m}$ de largura; trocanter curto, $80 \mu \mathrm{m}$; fêmur robusto, 420 $\mu \mathrm{m}$ de comprimento e $390 \mu \mathrm{m}$ de largura; tíbia, 360 $\mu \mathrm{m}$; tarso, $165 \mu \mathrm{m}$; unha curvada na ponta, $270 \mu \mathrm{m}$. Cerdas de todos os tamanhos; as "hair-like" podem alcançar 1,1 mm de comprimento. Região abdominal densamente coberta por cerdas curtas espiniformes que formam faixas. Região de maior concentração dos poros multiloculares. Poros multiloculares com dois anéis, sendo o mais externo com 15 a 17 lóculos e o mais interno quatro a oito lóculos (Fig. 11). Sete pares de espiráculos abdominais, sendo o primeiro submarginal. Dorso: corpo densamente coberto por cerdas médias e longas ("hair-like"). Ânus esclerotizado em forma de U. Poros multiloculares iguais ao do ventre.

Material examinado. BRASIL, Santa Catarina: Videira, (cistos), 4 espécimes em 3 lâminas, 08.VII.2008, M. L. Silva col., ex. raízes de Vitis sp.; Rio Grande do Sul: $ᄋ$, 2 espécimes em 2 lâminas, 10.I.1941(MRGC); Flores da Cunha, (+ , cistos, ninfas ambulatórias), 18 espécimes em 9 lâminas, 18.II.2009, V. Sganzerla col., ex. raízes de videira.; Bento Gonçalves, ㅇ, 2 espécimes em 2 lâminas, VIII.2000, I. Teixeira col., ex. Vitis vinifera (MRGC); + , 4 espécimes em 4 lâminas, 15.VIII.1999, I. Teixeira col., ex. videira; + , 6 espécimes em 6 lâminas, 15.X.2001, I. Teixeira col., ex. videira; (cistos), 8 espécimes em 3 lâminas, 05.XII.2008, D. C. Silva col., ex. raízes de videira; ㅇ, 7 espécimes em 7 lâminas, XI.2009, V. Sganzerla col., ex. raízes de videira; Caxias do Sul, 3 ㅇ, 1 lâmina, IV.2008, I. Onzi col., ex. videira; (cistos), 13 espécimes em 5 lâminas, 27.V.2008, D. A. Klering col., ex. videira.

Distribuição geográfica. Brasil (São Paulo, Paraná, Santa Catarina, Rio Grande do Sul) (Lepage, 1938; Silvestri, 1939; Lima, 1947; Vernalha, 1953; JAKUBSKI, 1965; SiLVA et al., 1968; BоTTON et al., 2000; Ben-Dov, 2005; Foldi, 2005).

Hospedeiros. Actinidiaceae: Actinidia deliciosa (quivizeiro); Amaryllidaceae: Amaryllis sp. (Amarílis); Aquifoliaceae: Ilex paraguariensis (erva-mate); Araucariaceae: Araucaria angustifolia (pinheirodo-Paraná); Arecaceae: Arecastrum romanzoffianum (palmeira); Asteraceae: Amphiachyris dracunculoides, Baccharis trimera (carqueja), Chaptalia nutans (arnica-do-campo), Chrysanthemum sp. (crisântemo), Cichorium endivia (almeirão), C. intybus (chicória-docafé), Dahlia sp. (dália), Gazania rigens (funcionária), Helianthus annuus (girassol), Helichrysum sp. (sempre-noiva), Lactuca sativa (alface), Matricaria chamomilla (camomila), Solidago microglossa (ervalanceta); Caricaceae: Carica papaya (mamoeiro); Caryophyllaceae: Dianthus caryophyllus (craveiro); Convolvulaceae: Convolvulus ottonis (cipó-de-veado), Ipomoea batatas (batata doce); Brassicaceae: Brassica campestris (mostarda), B. napus (nabo), Raphanus sativus (rabanete), R. raphanistrum (nabiça), Sinapis nigra; Cucurbitaceae: Citrullus vulgaris (melancia), Cucumis melo (melão), C. sativus (pepino), Cucurbita pepo (aboboreira), Lagenaria vulgaris (cabaça), Sechium 
edule (chuchuzeiro); Euphorbiaceae: Manihot palmata (aipim), M. utilissima (mandioca); Fabaceae: Arachis hypogea (amendoim), Glycine max (soja), Medicago sativa (alfafa); Fagaceae: Castanea vesca (castanheira portuguesa); Geraniaceae: Pelargonium zonale (gerânio); Juglandaceae: Juglans regia (nogueira), Carya illinoensis (nogueira pecã); Lamiaceae: Ocimum basilicum (manjericão), Rosmarinus officinalis (alecrim), Salvia officinalis (sálvia), S. splendens (alegria-dos-jardins); Malvaceae: Hibiscus esculentus (quiabeiro), Sida rhombifolia (vassoura, guaxuma); Meliaceae: Melia azedarach (cinamomo, lilás-daíndia); Moraceae: Ficus carica (figueira), Morus alba (amoreira branca), M. nigra (amoreira negra); Myrtaceae: Eucalyptus spp. (eucalipto), Feijoa sellowiana (goiabeira serrana), Psidium guajava (goiabeira); Onagraceae: Fuchsia sp. (brinco-deprincesa); Oxalidaceae: Oxalis articulata (azedinha), $O$. criorrhiza, O. sellowiana; Phytolaccaceae: Phytolacca decandra (caruru-bravo); Poaceae: Digitaria sp. (capimde-folha-larga), Saccharum officinarum (cana-deaçúcar), Zea mays (milho); Polygonaceae: Polygonum punctatum (erva-de-bicho), Rumex sp. (língua-de-vaca, azedinha); Portulacaceae: Talinum paniculatum (joãogomes); Punicaceae: Punica granatum (romãzeira); Rhamnaceae: Hovenia dulcis (uva-do-japão); Rosaceae: Cydonia oblonga (marmeleiro), Cydonia vulgaris (gamboeiro), Fragaria vesca (morangueiro silvestre), Malus domestica (macieira), Prunus spp. (ameixeira), Prunus avium (cerejeira), P. domestica (ameixeira preta), P. persica (pessegueiro), Pyrus communis (pereira), Rosa sp. (roseira); Rubiaceae: Gardenia jasminoides (gardênia); Salicaceae: Salix babylonica (salgueiro), S. humboldtiana (salseiro, chorão); Solanaceae: Solanum pseudoquina (quina branca), S. tuberosum (batatinha); Apiaceae: Apium graveolens (aipo), Daucus carota (cenoura), Petroselinum crispum (salsinha); Vitaceae: Vitis labrusca (videira americana), V. vinifera (videira européia); Zingiberaceae: Hedychium coronarium (lírio-do-brejo) (Costa, 1937; Lepage, 1938; Costa, 1941, 1944; Lima, 1947; Vernalha, 1953; Bertels, 1956; Fagundes et al., 1963; JAKUBSKi, 1965; Bertels \& BAUCKE, 1966; MARiconi \& ZAMith, 1973; Botton et al., 2000; BEN-Dov, 2005; Foldi, 2005).

Importância econômica. São pragas importantes de videira no sul do Brasil (Costa Lima, 1942; Costa, 1958; BotTon et al., 2000; Foldi, 2005).

\section{Eurhizococcus brevicornis (Silvestri, 1901)} (Fig. 12)

Termitococcus brevicornis SILVESTRI, 1901:5.

Eurhizococcus brevicornis; SILVESTRI, 1936:40.

Redescrição. Ninfa ambulatória ( $1^{\circ}$ instar). Corpo circular, 1,2 $\mathrm{mm}$ de comprimento e $1,0 \mathrm{~mm}$ de largura, coberto por cerdas de diferentes tamanhos; poros ausentes. Ventre: antena uniarticulada, com 113 a
$118 \mu \mathrm{m}$ e sete a oito cerdas curtas no ápice (Fig. 12). Espiráculos torácicos pequenos. Olhos ausentes. Longas cerdas marginais e submarginais em toda a extensão do corpo, sendo as marginais mais longas, podem alcançar até $315 \mu \mathrm{m}$ de comprimento; cerdas do corpo com aproximadamente $40 \mu \mathrm{m}$. Peças bucais desenvolvidas, estiletes muito longos e enrolados entre as pernas, podem formar até duas voltas completas. Pernas desenvolvidas e semelhantes em forma e tamanho: coxa globosa com duas cerdas; trocanter com duas sensilhas e uma pequena cerda; fêmur longo e robusto, com seis cerdas; tíbia e tarso delgados e semelhantes em tamanho; tíbia com um par de cerdas; tarso uniarticulado, com uma única cerda e um par de digítulos; unha levemente curvada. Pernas protorácicas (Fig. 12): coxa, $45 \mu \mathrm{m}$; trocanter curto, $25 \mu \mathrm{m}$; fêmur longo, com até $190 \mu \mathrm{m}$ de comprimento e $75 \mu \mathrm{m}$ de largura; tíbia, $75 \mu \mathrm{m}$; tarso, $73 \mu \mathrm{m}$; unha, $47 \mu \mathrm{m}$. Pernas mesotorácicas: coxa, $68 \mu \mathrm{m}$; trocanter, $25 \mu \mathrm{m}$; fêmur, $190 \mu \mathrm{m}$ de comprimento e $73 \mu \mathrm{m}$ de largura; tíbia, $78 \mu \mathrm{m}$; tarso, $76 \mu \mathrm{m}$; unha com até 52 $\mu \mathrm{m}$. Pernas metatorácicas: coxa, $75 \mu \mathrm{m}$; trocanter, 25 $\mu \mathrm{m}$; fêmur, $190 \mu \mathrm{m}$ de comprimento e $70 \mu \mathrm{m}$ de largura; tíbia, $90 \mu \mathrm{m}$; tarso, $82 \mu \mathrm{m}$; unha, $50 \mu \mathrm{m}$. Um par de cicatrizes metatorácicas com $16 \mu \mathrm{m}$, entre as coxas das pernas posteriores, que forma um eixo perpendicular em relação às mesmas. Cerdas caudais com até $420 \mu \mathrm{m}$. Sete pares de espiráculos abdominais, sendo o primeiro submarginal. Dorso: ânus esclerotizado em forma de U. Ninfa fixa $\left(2^{\circ}\right.$ instar - Cisto $)$. Desconhecida.

Fêmea adulta. Desconhecida.

Material examinado. Para preservar a estabilidade da nomenclatura desta espécie, um lectótipo é aqui designado entre os síntipos. Lectótipo (ninfa ambulatória), BRASIL, Mato Grosso: Cuiabá (Coxipo), sem outros dados, 2 espécimes em lâmina (IFSP); paralectótipos (ninfas ambulatórias), 1 espécime na mesma lâmina do lectótipo, e 3 espécimes em 2 lâminas distintas, mesmos dados do lectótipo (IFSP).

Distribuição geográfica. Brasil (Mato Grosso).

Biologia. Encontrada dentro do termiteiro de Capritermitis opaci parvi Silvestri (JAKUBSKI, 1965; MARICONI \& ZAMith, 1973; Ben-Dov, 2005).

\section{Eurhizococcus colombianus Jakubski, 1965}

(Figs 13-17)

Eurhizococcus colombianus JAKUBSKI, 1965:179.

Eurhizococcus brasiliensis; FigueroA Potes, 1946:216 (identificação errônea) Eurhizococcus silvestri JAKUBSKI, 1965:181 (nomem nudum).

Holótipo $\bigcirc$ (não examinado), COLÔMBIA, Rionegro, 13.XI.1944, E. J. Hambleton col., ex. raízes de Rubus glaucus, depositado no National Museum of Natural History, Washington D. C., USA (USNM) (BENDov, 2005).

Redescrição. Ninfa ambulatória ( $1^{\circ}$ instar). Corpo circular, com $885 \mu \mathrm{m}$ de comprimento e $815 \mu \mathrm{m}$ de largura e coberto por cerdas de diferentes tamanhos; ausência de poros. Ventre: antena com dois artículos, sendo o último 


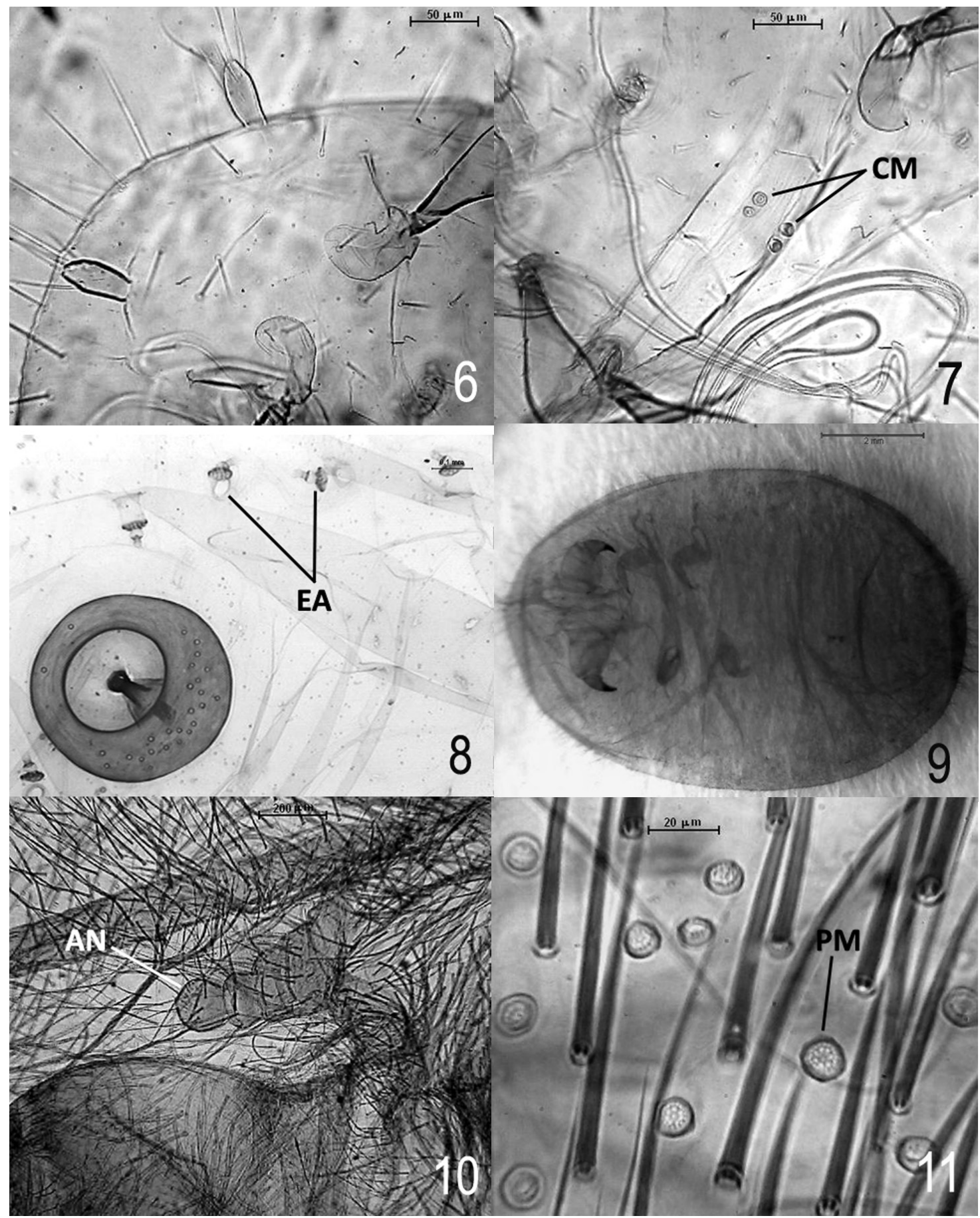

Figs 6-11. Eurhizococcus brasiliensis, ninfa ambulatória: 6, cabeça, antenas; 7, cicatrizes metatorácicas; 8, cisto: placa anal, espiráculos abdominais; . : 9, vista ventral; 10, antena; 11, poros multiloculares (AN, antena; CM, cicatrizes metatorácicas; EA, espiráculos abdominais; PM, poros multiloculares).

$(135 \mu \mathrm{m})$ nove vezes maior que o primeiro $(15 \mu \mathrm{m})$; último artículo em forma de clava, com seis cerdas (Fig. 13). Espiráculos torácicos pequenos. Olhos ausentes. Longas cerdas marginais que podem alcançar até $425 \mu \mathrm{m}$ de comprimento; cerdas caudais diferenciadas ausentes; cerdas do corpo com até $85 \mu \mathrm{m}$ de comprimento. Peças bucais desenvolvidas, estiletes muito longos, podem dar até duas voltas completas. Pernas desenvolvidas e semelhantes em forma e tamanho: coxa globosa; trocanter com duas sensilhas e uma cerda curta; fêmur longo e robusto, com cinco cerdas; tarso uniarticulado e levemente maior que a tíbia, ambos com uma cerda; unha levemente curvada. Pernas protorácicas: coxa, $90 \mu \mathrm{m}$; trocanter, $40 \mu \mathrm{m}$; fêmur, $170 \mu \mathrm{m}$ de comprimento e 70 $\mu \mathrm{m}$ de largura; tíbia, $78 \mu \mathrm{m}$; tarso, $95 \mu \mathrm{m}$; unha, $60 \mu \mathrm{m}$. Pernas mesotorácicas: $110 \mu \mathrm{m}$; trocanter, $38 \mu \mathrm{m}$; fêmur, $173 \mu \mathrm{m}$ de comprimento e $70 \mu \mathrm{m}$ de largura; tíbia, 77 $\mu \mathrm{m}$; tarso, $100 \mu \mathrm{m}$; unha, $68 \mu \mathrm{m}$. Pernas metatorácicas: coxa, $110 \mu \mathrm{m}$; trocanter, $40 \mu \mathrm{m}$; fêmur, $190 \mu \mathrm{m}$ de comprimento e $67 \mu \mathrm{m}$ de largura; tíbia, $90 \mu \mathrm{m}$; tarso, 120 


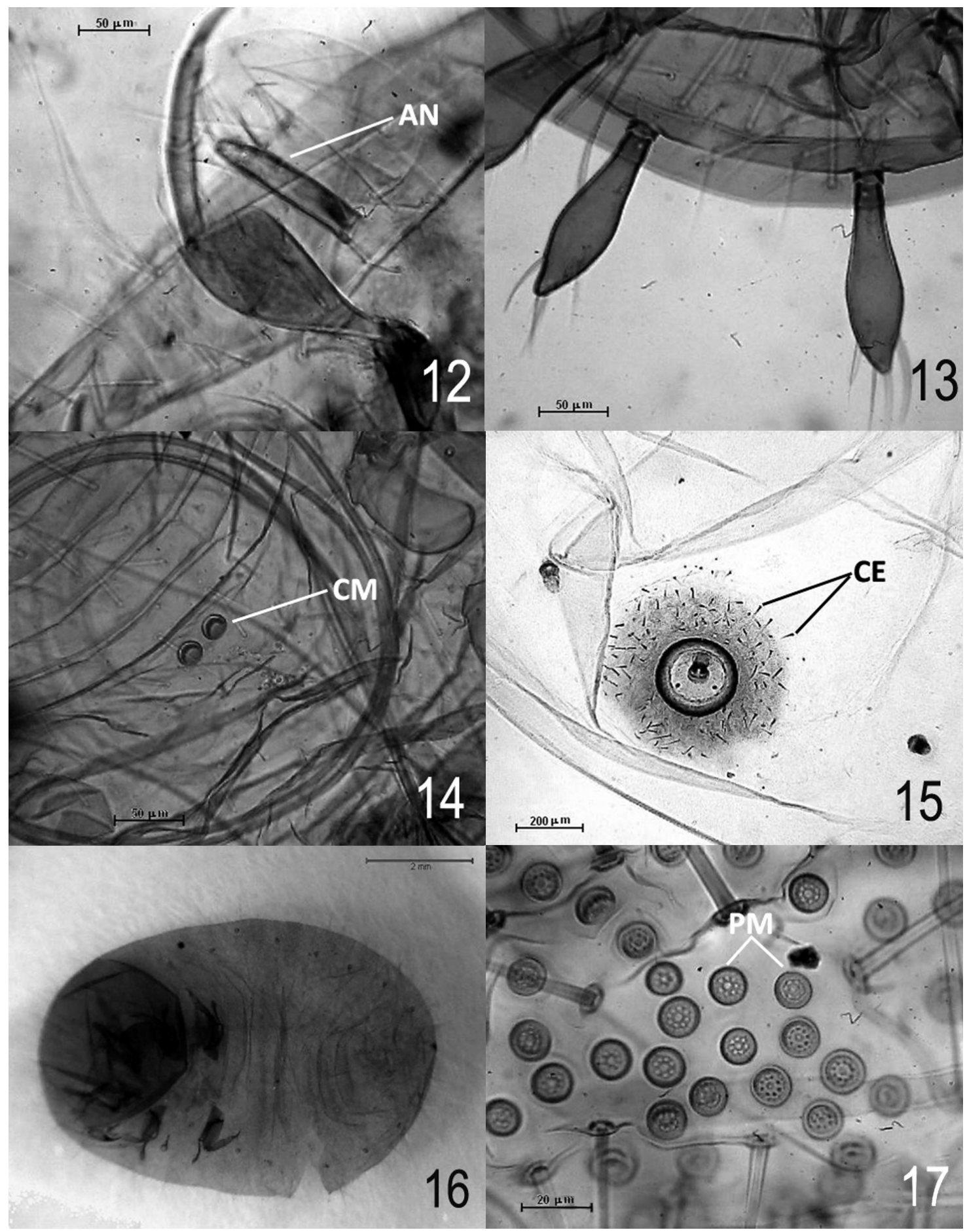

Figs 12-17. 12, Eurhizococcus brevicornis, ninfa ambulatória: antena e perna protorácica direita; Eurhizococcus colombianus, ninfa ambulatória: 13, antenas; 14, cicatrizes metatorácicas; 15 , cisto: visão geral da placa anal; + : 16, vista ventral; 17, poros multiloculares (AN, antena; CE, cerdas espiniformes; CM, cicatrizes metatorácicas; PM, poros multiloculares).

$\mu \mathrm{m}$; unha, $75 \mu \mathrm{m}$. Um par de cicatrizes metatorácicas com $19 \mu \mathrm{m}$, entre as coxas posteriores formam um eixo paralelo em relação às mesmas (Fig. 14). Pequenas cicatrizes cruciformes em toda a margem posterior do corpo. Sete pares de espiráculos abdominais, o primeiro submarginal, o segundo e terceiro pares maiores que os demais. Dorso: ânus esclerotizado em forma de U.

Ninfa fixa ( $2^{\circ}$ instar - Cisto). Corpo oval ou suboval, com 5,4 $\mathrm{mm}$ de comprimento e $5,0 \mathrm{~mm}$ de largura (na parte posterior). Derme alveolar. Ventre: antena pequena e circular $(24 \mu \mathrm{m})$, com quatro cerdas. Olhos e pernas ausentes. Peças bucais desenvolvidas. Conjunto de cicatrizes (até 28) ao redor das peças bucais. Poros periespiraculares presentes nos espiráculos torácicos: até dez poros nos espiráculos anteriores e quatro nos posteriores. Dorso: sete pares de espiráculos 
abdominais, o primeiro submarginal e o último um pouco menor que os demais. Ânus em forma de U circundado por uma placa circular esclerotizada que forma dois anéis - o mais externo com até $120 \mu \mathrm{m}$ de diâmetro. Ao redor desta placa, inúmeras cerdas pequenas, similares a espinhos (Fig. 15).

Fêmea adulta. Corpo alongado com $8,5 \mathrm{~mm}$ de comprimento e $5,0 \mathrm{~mm}$ de largura, densamente coberto por cerdas longas, médias e curtas; segmentação claramente visível; presença de poros multiloculares. Ventre (Fig. 16): antena com seis artículos - I, $81 \mu \mathrm{m}$; II, $87 \mu \mathrm{m}$; III, $56 \mu \mathrm{m}$; IV e V, $81 \mu \mathrm{m}$ cada; VI, alongado, com $150 \mu \mathrm{m}$ de comprimento e 12 cerdas. Entre um artículo e outro da antena, uma fileira de pequenas cerdas espiniformes. Olhos e peças bucais ausentes. Pernas desenvolvidas, o primeiro par robusto do tipo fossorial, adaptado para cavar o solo. Pernas protorácicas: coxa globosa; trocanter e fêmur fusionados, com $570 \mu \mathrm{m}$ de comprimento e 585 $\mu \mathrm{m}$ de largura; fêmur grande e robusto; tíbia com $113 \mu \mathrm{m}$ de comprimento; tarso e unha fusionados dorsalmente, $450 \mu \mathrm{m}$; unha curvada, $385 \mu \mathrm{m}$ de comprimento, desenvolvida e esclerotizada. Pernas mesotorácicas: coxa globosa; trocanter pequeno, $130 \mu \mathrm{m}$; fêmur longo, $490 \mu \mathrm{m}$ de comprimento e $300 \mu \mathrm{m}$ de largura; tíbia, $340 \mu \mathrm{m}$; tarso, $190 \mu \mathrm{m}$; unha curvada, $310 \mu \mathrm{m}$. Pernas metatorácicas: coxa globosa; trocanter pequeno, 140 $\mu \mathrm{m}$; fêmur longo, $540 \mu \mathrm{m}$ de comprimento e $300 \mu \mathrm{m}$ de largura; tíbia, $370 \mu \mathrm{m}$; tarso, $200 \mu \mathrm{m}$; unha curvada, 270 $\mu \mathrm{m}$. Cerdas longas ("hair-like") cobrem densamente o corpo, principalmente as margens. Cerdas médias e curtas do tipo espiniformes cobrem densamente todo o corpo, principalmente as porções finais do abdômen onde há maior concentração também dos poros multiloculares. No abdômen, as cerdas curtas do tipo espiniforme formam bandas ao longo de cada segmento abdominal. Poros multiloculares com dois anéis: o mais externo com 16 a 20 lóculos e o mais interno cinco a sete lóculos (Fig. 17). Espiráculos torácicos desenvolvidos, com poros no átrio. Sete pares de espiráculos abdominais, o primeiro submarginal. Átrio dos espiráculos abdominais com poros multiloculares. Dorso: corpo densamente coberto por cerdas. Ânus esclerotizado em forma de U. Poros multiloculares iguais aos do ventre.

Material examinado. COLÔMBIA, Antioquia: Rionegro, , espécime em lâmina, 21.V.2008, M. E. Londoño col., ex. raízes de Rubus glaucus (UCDC); (ninfas ambulatórias), 4 espécimes em 2 lâminas, 21.V.2008, M. E. Londoño col., ex. raízes de Rubus glaucus (UCDC); (cistos), 2 espécimes em 2 lâminas, 21.V.2008, M. E. Londoño col., ex. raízes de Rubus glaucus (UCDC).

Distribuição geográfica. Colômbia (Antioquia, Valle del Cauca).

Hospedeiros. Rosaceae: Rubus glaucus (framboesa); Umbelliferae: Arracacia xanthorhiza (mandioquinha salsa); Vitaceae: Vitis labrusca (videira) (Kondo, 2001; Ben-Dov, 2005; Kondo \& Gómez, 2008).
Agradecimentos. Aos curadores e responsáveis pelas coleções e empréstimo de material: E. Guerieri (IFSP), C. Campaner (MZSP) e P. Gullan (UCDC); Dr. Takumasa Kondo (CORPOICA), pela atenção e sugestões dadas ao trabalho; à FEPAGRO, pela utilização do laboratório e do Museu de Entomologia (MRGC) e à Dra. Vera Wolff (FEPAGRO), pela orientação, companheirismo, amizade e incentivo.

\section{REFERÊNCIAS BIBLIOGRÁFICAS}

BEN-DOv, Y. 2005. A systematic catalogue of the scale insect Family Margarodidae (Hemiptera: Coccoidea) of the world. Wimborne, Intercept Limited. 400p.

Bertels, A. 1956. Entomologia agrícola sul-brasileira. Rio de Janeiro, Ministério Agricultura (Série didática n. 16). 458p.

Bertels, A. \& Bauke, O. 1966. Segunda relação das pragas das plantas cultivadas no RS. Pesquisa Agropecuária Brasileira 1:17-46.

Botton, M.; Hickel, E. R.; Soria, S. J. \& TeiXeira, I. 2000. Bioecologia e controle da Pérola-da-terra Eurhizococcus brasiliensis (Hempel, 1922) (Hemiptera: Margarodidae) na cultura da videira. Bento Gonçalves, Embrapa (Circular Técnica n. 27). 23p.

Costa, R. G. 1937. Mais uma contribuição para o estudo do Margarodes brasiliensis Hemp. Revista Agronômica 1(7):336-338.

1941. Pragas das plantas cultivadas no Rio Grande do Sul. Revista Agronômica 5(60):717-719.

. 1944. Pragas das plantas cultivadas do Rio Grande do Sul. Porto Alegre, Secretaria do Estado dos Negócios da Agricultura, Indústria e Comércio. 136p.

1958. Alguns insetos e outros pequenos animais que danificam plantas cultivadas no RS. Porto Alegre, Secretaria de Agricultura, Indústria e Comércio, (S.I.P.A. 172, Série A). 296p.

Costa Lima, A. 1924. Sobre insectos parasitas da videira. Almanak Agricola Brasileiro 1924:135-141.

1942. Insetos do Brasil. Homópteros. Rio de Janeiro, Escola Nacional de Agronomia, Tomo 3 (Série didática no 4). 327p. 1950 (1949). Nova espécie do Margarodes do Brasil (Coccoidea, Margarodidae). Memórias do Instituto Oswaldo Cruz 47(1-2):241-248.

Fagundes, A. C.; Redaeli, D. C.; Corseuil, E.; Mata, N. G.; Baucke, O. \& Fagundes, R. G. 1963. Pragas da Agricultura do Rio Grande do Sul. Porto Alegre, Serviço de Entomologia, Diretoria da Produção Vegetal, Secretaria da Agricultura.

Fernald, M. E. 1903. A catalogue of the Coccidae of the world. Bulletin of the Hatch Experiment Station of the Massachusetts Agricultural College 88:1-360

Figueroa Potes, A. 1946. Catalogación inicial de las cochonilhas del Valle del Cauca (Homoptera - Coccoidea) Revista Facultad de Agronomia, Montevideo Universidad 6:196-220.

FoldI, I. 1987. Une cochenille, Margarodes soriai, n. sp. nuisibles à lavigne au Brésil (Homoptera, Coccoidea, Margarodidae). Annales de la Société Entomologique de France 23(2):155-160. 1989. Note sur l'identité de Margarodes soria Foldi, 1987 (Homoptera, Coccoidea, Margarodidae). Nouvelle Revue d'Entomologie 6(3):320.

.2001. A world list of extant and fossil species of Margarodidae sensu lato (Hemiptera: Coccoidea). Nouvelle Revue d'Entomologie 18(3):195-231.

2005. Ground pearls: a generic revision of the Margarodidae sensu stricto (Hemiptera: Sternorhyncha: Coccoidea) Annales de la Société Entomologique de France 41(1):81-125.

Granara de Willink, M. C. 1990. Conociendo nuestra fauna. I. Superfamilia Coccoidea (Homoptera: Sternorrhyncha). San Miguel de Tucumán, Facultad de Ciencias Naturales y Instituto Miguel Lillo (Série Monográfica y Didáctica $\mathrm{n}^{\underline{0}}$ 6). 43p.

1995. Conociendo nuestra fauna VIII. Familias Margarodidae y Ortheziidae (Homoptera: Coccoidea). San Miguel de Tucumán, Facultad de Ciencias Naturales y Instituto Miguel Lillo (Série Monográfica y Didáctica $\mathrm{n}^{\circ}$ 26). 19p.

JAKUBSKI, A. W. 1965. A critical revision of the families Margarodidae and Termitococcidae (Hemiptera, Coccoidea). London, Trustees of the British Museum (Natural History). 187p.

Kondo, T. 2001. Las Cochinillas de Colombia (Hemiptera: Coccoidea). Biota Colombiana 2(1):31-48.

Kondo, T. \& Gómez, C. E. 2008. La Perla de Tierra, Eurhizococcus colombianus Jakubski, uma nueva plaga de la vid, Vitis labrusca L. en el Valle Del Cauca, Colombia. Novedades Técnicas 10:34-40. 
Lepage, H. S. 1938. Catálogo dos coccídeos do Brasil. Revista do Museu Paulista 23:327-491.

Lima, A. D. F. 1947. Insetos fitófagos de Santa Catarina. Boletim Fitossanitário 2(3-4):233-251.

Lindinger, L. 1943. Verzeichnis der Schildlaus-Gattungen, 1. Nachtrag. (Homoptera: Coccoidea). Zeitschrift der Wiener Entomologischen Gesellschaft 28:205-208; 217-224; 264-265.

MacGillivray,A. D. 1921. The Coccidae. Tables for the identification of the subfamilies and some of the more important genera and species, together with discussions of their anatomy and life history. Urbana, III: Scarab Company. 502p.

Mariconi, F. A. M. \& ZAMith, A. P. L. 1973. Contribuição para o conhecimento dos Margarodinae (Homoptera, Margarodidae) que ocorrem no Brasil. Anais da Sociedade Entomológica do Brasil 2(1):86-101.

Miller, D. R.; Urng, A.; Venable, G. L. \& Gill, R. J. 2007. Systematic Entomology Laboratory, BARC, USDA. Disponível em: $<$ http://www.sel.barc.usda.gov/ ScaleKeys/ScaleInsectsHome/ ScaleInsectsHome.html>. Acesso em: 09.05.2008.

Morrison, H. 1928. A classification of the higher groups and genera of the coccid family Margarodidae. Washington D. C., United States Department of Agriculture (Technical Bulletin n. 52). 239p.

Silva, A. G. D.; Gonçalves, C. R.; Galvẽo, D. M.; Gonçalves, A. J. L.; Gomes, J.; Silva, M. N. \& Simoni, L. 1968. Quarto catálogo dos insetos que vivem nas plantas do Brasil. Seus parasitos e predadores. Parte II, $1^{\circ}$ Tomo - Insetos, Hospedeiros e Inimigos Naturais. Rio de Janeiro, Ministério da Agricultura. 622p.

Silvestri, F. 1901. Descrizione di nuovi termitofili e relazioni di essi com gli ospiti. II. Hemiptera, Homoptera, Fam. Coccidae. Bolletino del Musei di Zoologia ed di Anatomia Comparata 16:4-6.

. 1936. Ridescrizione del genere Termitococcus Silv. con una specie nuova del Brasile e descrizione di un nuovo genere affine. Bolletino del Laboratorio di Zoologia Generale e Agraria 30:32-40.

. 1939. Compendio di Entomologia Applicata. Parte speciale. Portici, Tipografia Bellavista. 974p.

Vernalha, M. M. 1953. Coccídeos da coleção I.B.P.T. Arquivos de Biologia e Tecnologia 8:111-304.

Wille, J. 1922. Margarodes brasiliensis [n. sp.]. Egatea 7:83-85.

Recebido em 2 de abril de 2012. Aceito em 30 de agosto de 2012. ISSN 0073-4721

Artigo disponível em: www.scielo.br/isz 